\title{
The influence of corrosion phenomena on operational parameters of modern fuel injectors CI-engines
}

The article describes the influence of corrosion phenomena on operational parameters of common fuel injectors. Paper has been presented and describes Common Rail fuel injectors, their structure and work principles. Main fuel injectors parameters, like injection dosage, back leakage and injection delay are discussed. It has been presented the elements of injectors which have influence on dosages magnitude and how the corrosion phenomena influences on its. The elements of injectors, which have an influence on dosages magnitudes, are presented and the effect of their corrosion is discussed.

Key words: corrosion phenomena, fuel dosages, Common Rail system, fuel injector

\section{Introduction}

Corrosion phenomena is an undesirable process of destroying materials. This process is driven by a combination of chemical, electrochemical, microbiological and mechanical factors. The results of corrosion phenomena on mechanical objects is increased wear, which has a major impact on working parameters [2] of fuel injectors. Harsh environmental conditions, like extreme pressures, temperatures and fuel moisture that are encountered by fuel injectors accelerate this deteriorating effect [8]. Since all fuel injectors are made from a tool steel (both electromagnetic and piezoelectric injectors share this property) the choice of material or injector type does affect the speed of corrosion, and unfavorable environmental conditions remain a primary driver for corrosion. The corrosion types are mainly: pit, expansion joint corrosion and specific aspect corrosion in moving medium in fuel injectors. Pit corrosion is the form of located medium fit in results appearing local loss material - pits. Damages trigger off pit corrosion on precise elements make the leaks. Intensive local corrosion in covered places or slats (cracks) metal elements expose on acting medium is expansion joint corrosion [4]. The main factor which makes this type of corrosion is unequal access to oxidant. It appears under seals, washers and gaskets in fuel injectors. Pit and expansion joint corrosion are sometimes compared and treated as the same type. The mechanism is similar but the appearing reason of expansion joint corrosion are slats or crack and pit corrosion appears themselves. The corrosion in moving medium depends on the speed of flowing liquid. It is examined for specific conditions: laminar flow, turbulent flow and cavitation corrosion. Liquid flow influences the speed of corrosion. In addition the corrosion is accompanied by metal erosion phenomena. Connecting the effects of mechanic liquid impact on metal called erosion corrosion. Therefore it can be said that the corrosion phenomena is increased by erosion, which in turn depends on the speed of liquid flow and temperature inside the fuel injector [4].

Turbulent fuel flow increases the damaging effect on injector elements. Its parts corrode quickly when faced against an intensive flow, which exceeds a certain speed threshold.
Fast flow through variable cross-section may lead to cavitation, which is the formation of vapor cavities in areas, where local pressure drops. When such cavity experiences a pressure-increase it implodes, generating an intensive shockwave, which damages metallic surfaces. This effect is called a cavitation corrosion, and is frequently encountered in fuel injectors [2, 4].

The last sort of corrosion that occurs in fuel injectors is friction corrosion. It mainly appears in precise elements because of a sliding motion of these elements. Figure 1 presents the sort of corrosion phenomena in fuel injectors.

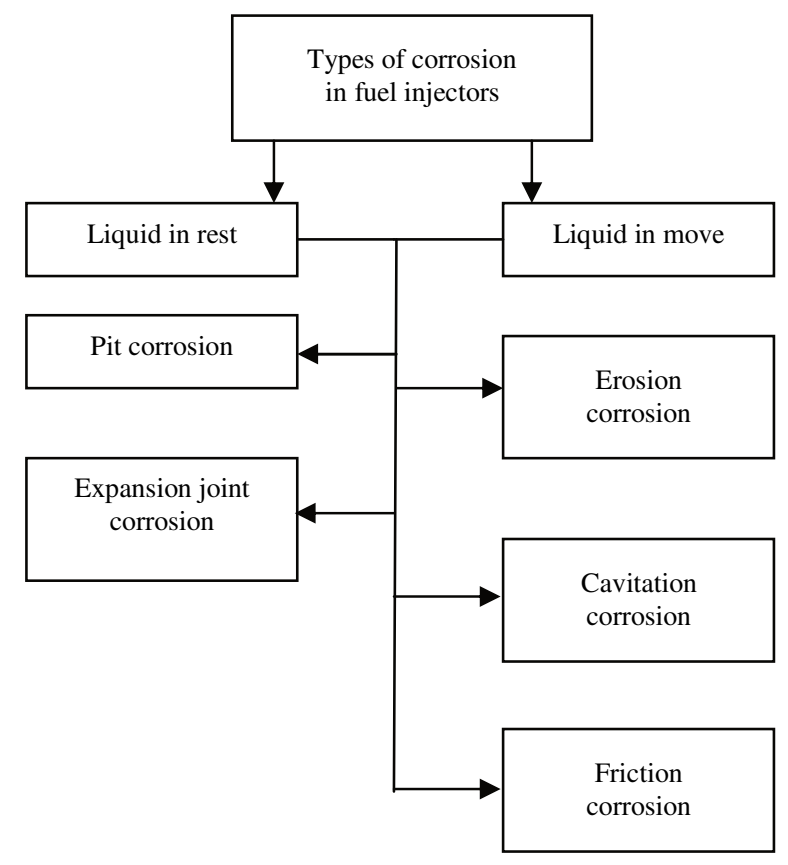

Fig. 1. Types of corrosion in fuel injectors

\section{Analysis of fuel injector operational parameters}

Fuel injector is a device which deliver fuel to combustion chamber CI-engine. Its main task is to supply an engine's combustion chamber with a specific fuel dosage under given pressure. So injector is responsible for spray and fuel distribution. Modern CI-engines are equipped with Common Rail system. This solution separates the work of pressure pump and fuel injectors through high pressure 
accumulator. It allows steering of system pressure, injection time and timing $[3,5]$.

The fuel injectors parameters could be divide into operational and diagnostic. Operational parameters directly influence CI-engine work. These are injection and return dosages. Diagnostic parameters allow to determine technical condition of fuel injector. Figure 2 presents the research protocol of piezoelectric Continental VDO Siemens fuel injector on Zapp CRU2i test bench.

\begin{tabular}{|c|c|c|c|c|c|c|}
\hline \multicolumn{3}{|l|}{$\begin{array}{l}\text { Zlecenie: c } 30 \\
\text { INJ: VDO, CR, 5WS } 40677, \text { PIEZO } \\
\text { SN: } 1-5\end{array}$} & \multicolumn{4}{|c|}{$\begin{array}{r}\text { Wynik } \\
\text { protokolu } \\
:\end{array}$} \\
\hline$\underset{H P}{\text { LKT }}=1600,180 "$ (g/min) (R) & $\begin{array}{r}0 \\
65\end{array}$ & 2.81 & & & & 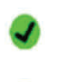 \\
\hline eRLC & $\begin{array}{l}2.20 \\
7.10\end{array}$ & 3.30 & & & & 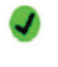 \\
\hline IVM VTP. 1 (mg/STRK) (D) & $\begin{array}{l}41.12 \\
53.12\end{array}$ & 23.06 & (R) & $\begin{array}{r}0.00 \\
37.23\end{array}$ & 19.39 & $x$ \\
\hline iVM VTP.2 (mg/stRK) (D) & $\begin{array}{l}1,47 \\
12.47\end{array}$ & 1.45 & & & & $x$ \\
\hline $\begin{array}{l}\text { STRK: } 1000 \text {, HP: } 1250 \mathrm{bar}, \mathrm{P} / \mathrm{STRK} \\
\text { iVM VTP. } 3 \text { (mg/STRK) (D) } \\
\text { STRK: } 1000 \text {, } \mathrm{HP}: 850 \mathrm{bar}, \mathrm{P} / \mathrm{STRK} \text { : }\end{array}$ & $\begin{array}{l}: 1, \text { SU } \\
17.19 \\
29.19 \\
1, \text { SUN }\end{array}$ & $\begin{array}{l}\mathrm{JM}[\mathrm{P}]: 29 \\
8.31 \\
\mathrm{~A}[\mathrm{P}]: 440\end{array}$ & Ousec & & & $x$ \\
\hline $\begin{array}{l}\text { iVM VTP.4 (mg/STRK) (D) } \\
\text { STRK: } 1000 \text {, HP: } 240 \text { bar, P/STRK: }\end{array}$ & $\begin{array}{l}7.31 \\
12.31 \\
1, \text { SUN }\end{array}$ & $\begin{array}{l}2.77 \\
{[\mathrm{P}]: 510}\end{array}$ & & & & $x$ \\
\hline NOP & $\begin{array}{l}120 \\
190\end{array}$ & 120.00 & & & & $\lambda$ \\
\hline RSP & $\begin{array}{l}200 \\
420\end{array}$ & 369.00 & & & & $\Omega$ \\
\hline
\end{tabular}

Fig. 2. The protocol of research fuel injector on Zapp CRU $2 \mathrm{i}$ test bench

The operational parameters are VTP $1-4$ injection dosages and return dosage by VTP 1 test. VTP $1-4$ are the types of dosages. VTP 1 is a full load dosage under 160 $\mathrm{MPa}$ system pressure within $1020 \mu \mathrm{s}$. This mensuration is at maximum engine load and it also includes return dosage Correct return dosage at maximum load guarantees, that it will stay correct for all other pressure levels, therefore it is not measured in tests VTP.2-4.

The VTP 2 is an initial dosage test. This test is performed under $125 \mathrm{MPa}$ pressure and low injection time of $290 \mu \mathrm{s}$. This is very important dosage and regulate is as the first, because depend from range of movement steering valve.

VTP 3 is a middle load dosage under $85 \mathrm{MPa}$ system pressure and $440 \mu$ s injection time. The last VTP 4 is idle speed dosage under $24 \mathrm{MPa}$ pressure and $510 \mu$ s injection time. It should be noted that the difference between all injectors on engine in this dosage amount $0.5 \mathrm{mg} / \mathrm{STRK}$. If higher differences would be encountered the engine would work unsteadily.

Figure 2 also depicts diagnostic parameters. LKT is an injector leak test in rest. This is very important parameter, since a condition of inner sealing and precision elements can be determine base on its analysis. Only back leakage was measured during this test. Test eRLC is an electrical test. Electrical capacity, insulation resistance between pins by $100 \mathrm{~V}$ and ground resistance by $250 \mathrm{~V}$ in piezoelectric injectors are measured. NOP is nozzle open pressure test. This parameter measures what level of pressure opens injector nozzle. RSP is delay fuel injection parameter. This is a time between steering electrical element and starting spraying [1, 6, 7]. Figure 3 presents fuel injector diagnostic parameters. Entrances parameters are injection pressure, time and frequency. Output parameters are injection return dosages, injection delay, nozzle open pressure and temperature. Temperature involves injection and return fuel temperature and injector body temperature. This parameter is very important, because it shows the area of injector damage.

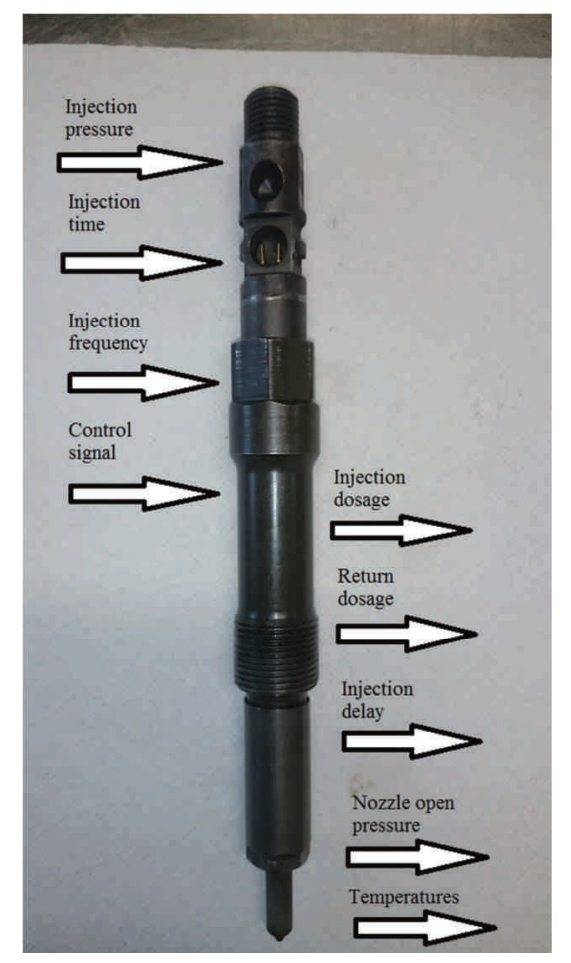

Fig. 3. Fuel injector diagnostic parameters

\section{Presentation of test stand}

Tests have been performed on tests bench Zapp CRU $2 \mathrm{i}$ (Fig. 4), which allowed for measurements of the following parameters:

- fuel injector delay,

- nozzle open pressure,

- injection and return dosages,

- electric parameters.

Research of the effects of corrosion were carried out using the FL150/70 laboratory microscope (Fig. 5).

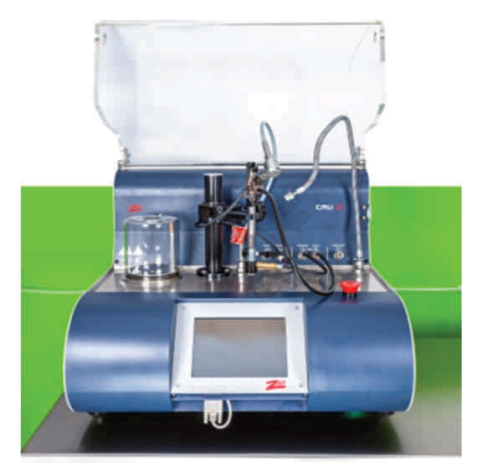

Fig. 4. Zapp CRU 2i test bench 


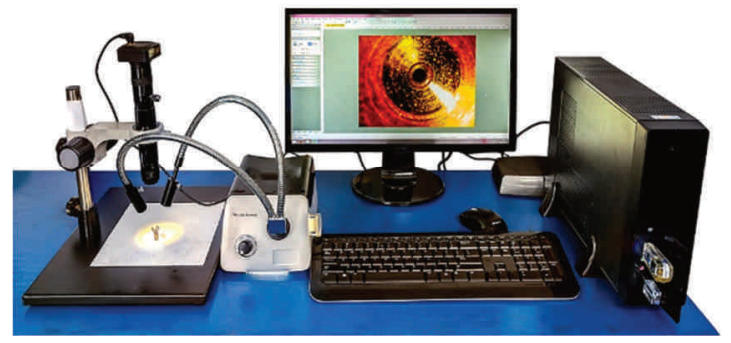

Fig. 5. Laboratory microscope FL150/70

\section{Test results}

Corrosion phenomena are examined in two aspects:

- when the fuel injector is out of order and needs to be repaired,

- when the fuel injector works but its operating parameters are not correct.

In the first case there are many signs of corrosion, especially on precision elements. Figures $6-8$ present the corroded parts of Bosch fuel injector.

A

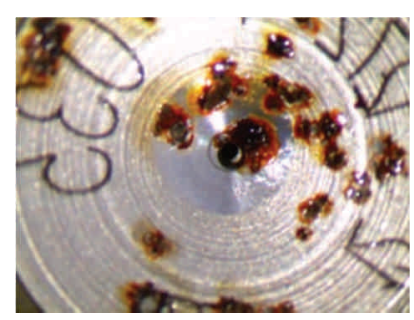

Fig. 6. A-steering valve, B - not working part of the needle

A

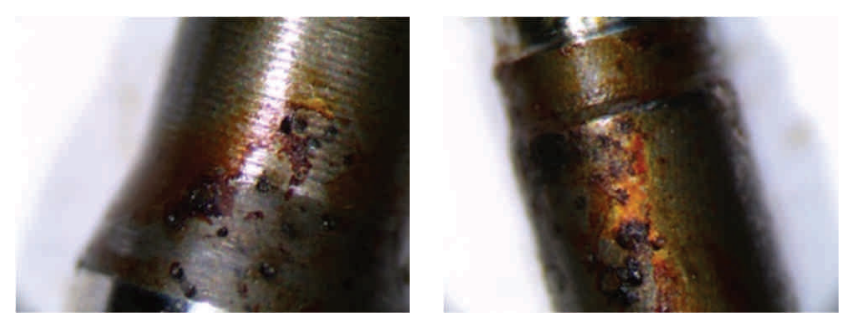

Fig. 7. A - not working part of the injector piston, $\mathrm{B}-$ precision element of the injector piston

A

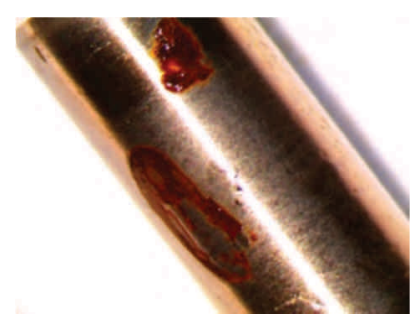

Fig. 8. A - precision element of injector needle, B - sealing surface on injector body between injector nozzle and injector body

Damages due to corrosion, which are presented in Figs 6-8, qualify elements to replacement. Fuel injector valve presented in Fig. 6 is damaged and due to pits it lost tightness. Not working parts of injector's needle and piston are very corroded (Figs 6, 7). Observations point to the conclusion that a quality of the fuel was of poor quality, with large amount of moisture. Precision element of injector needle (Fig. 8) has been effaced and polluted. There are methods depending on shining precision element but not by so huge damages. These defects influence the fuel injectors operational parameters, which became leaky against return dosages, what is easily observed on the test bench Problem begins when engine work but unsteadily in middle load when there is no correction capacity to read. It may be cause because of huge difference between injection dosages.
A

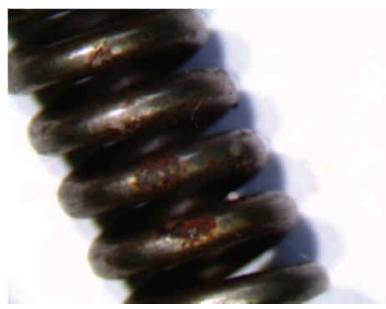

B

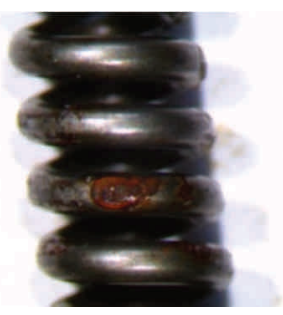

Fig. 9. A - injector needle spring, B - spring in electromagnetic solenoid
A

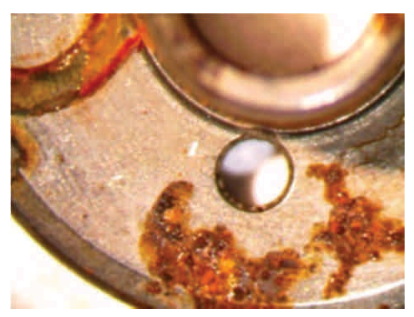

B

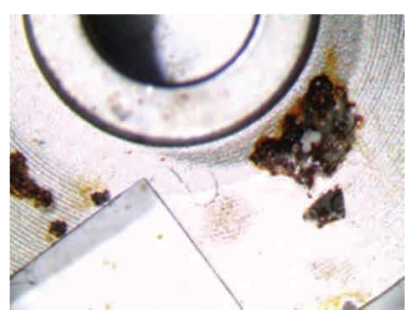

Fig. 10. A - elements of electromagnetic system valve, B - elements of electromagnetic system plate
A

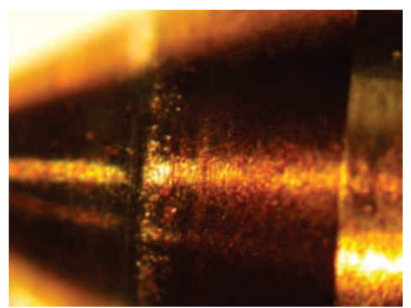

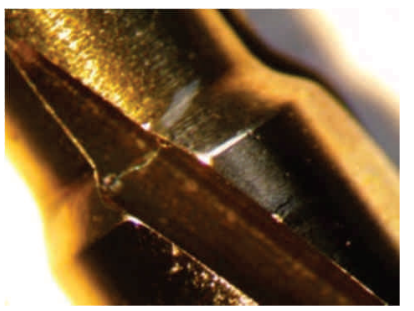

Fig. 11. A - corroded fuel injector needle, B - fuel intake channels damaged by cavitation corrosion in Delphi injectors

Figure 9 A presents injector needle spring with visible corrosion, which affects operational characteristics of the injector and has a direct impact on injection dosages. Nevertheless it is still possible to regulate dosages through washers over injector needle. Spring in the solenoid depends on the return dosage. Results show that decreasing the thickness of the solenoid's washer by $20 \%$ doubles the return dosage. Corroded injector valve elements (Fig. 10) could change fuel injector work parameters like injection dosages, delay nozzle open pressure. Figure 11 presents the corroded fuel injector needle and damaged fuel intake channels in Delphi injectors caused by cavitation.

Although the tested injector still works, all presented symptoms lead to deterioration of operational parameters of the injector and have a non-negligible impact on its performance. 
Table 1. Test results of good working electromagnetic Bosch fuel injector research

\begin{tabular}{|c|c|c|c|c|}
\hline \multicolumn{5}{|c|}{ Electromagnetic Bosch fuel injector no. 0445110320} \\
\hline eRLC & \multicolumn{2}{|c|}{$\begin{array}{c}\text { Resistance } \\
{[0.3-0.7 \Omega]}\end{array}$} & \multicolumn{2}{|c|}{$\begin{array}{c}\text { Inductance } \\
{[115-375 \mu \mathrm{H}]}\end{array}$} \\
\hline & \multicolumn{2}{|c|}{0.4} & \multicolumn{2}{|c|}{294} \\
\hline RSP & \multicolumn{2}{|c|}{$\begin{array}{l}\text { Injection delay } \\
{[200-500 \mu \mathrm{s}]}\end{array}$} & & \\
\hline & \multicolumn{2}{|c|}{434} & & \\
\hline \multirow[t]{2}{*}{ NOP } & \multicolumn{2}{|c|}{$\begin{array}{c}\text { Nozzle open pressure } \\
{[14-21 \mathrm{MPa}]}\end{array}$} & & \\
\hline & \multicolumn{2}{|c|}{19} & & \\
\hline Test & \multicolumn{2}{|c|}{ Injection dosage } & \multicolumn{2}{|c|}{ Return dosage } \\
\hline $\begin{array}{l}\text { Leak test }\left[\mathrm{mm}^{3} / \mathrm{H}\right] \\
170 \mathrm{MPa}, 300 \mathrm{~s}\end{array}$ & \multicolumn{2}{|c|}{0} & $\begin{array}{c}0-80 \\
{[300 \mathrm{~s}]}\end{array}$ & 9.44 \\
\hline $\begin{array}{l}\text { IVM } 1\left[\mathrm{~mm}^{3} / \mathrm{H}\right] \\
160 \mathrm{MPa}, 800 \mu \mathrm{s}\end{array}$ & $41.16-52.4$ & 44.72 & $5-82$ & 28,59 \\
\hline $\begin{array}{l}\text { IVM } 2\left[\mathrm{~mm}^{3} / \mathrm{H}\right] \\
60 \mathrm{MPa}, 630 \mu \mathrm{s}\end{array}$ & $8-15.68$ & 11.06 & & \\
\hline $\begin{array}{l}\text { IVM } 3\left[\mathrm{~mm}^{3} / \mathrm{H}\right] \\
60 \mathrm{MPa}, 640 \mu \mathrm{s}\end{array}$ & $0.88-6.48$ & 3.60 & & \\
\hline $\begin{array}{l}\text { IVM } 4\left[\mathrm{~mm}^{3} / \mathrm{H}\right] \\
60 \mathrm{MPa}, 275 \mu \mathrm{s}\end{array}$ & $0.24-2.96$ & 0.84 & & \\
\hline
\end{tabular}

Table 2. Test results of faulty electromagnetic Bosch fuel injector research

\begin{tabular}{|c|c|c|c|c|}
\hline \multicolumn{5}{|c|}{ Electromagnetic Bosch fuel injector no. 0445110320} \\
\hline eRLC & \multicolumn{2}{|c|}{$\begin{array}{c}\text { Resistance } \\
{[0.3-0.7 \Omega]}\end{array}$} & \multicolumn{2}{|c|}{$\begin{array}{c}\text { Inductance } \\
{[115-375 \mu \mathrm{H}]}\end{array}$} \\
\hline & \multicolumn{2}{|c|}{0.4} & \multicolumn{2}{|c|}{294} \\
\hline RSP & \multicolumn{2}{|c|}{$\begin{array}{c}\text { Injection delay } \\
{[200-500 \mu \mathrm{s}]}\end{array}$} & & \\
\hline & \multicolumn{2}{|c|}{674} & & \\
\hline \multirow[t]{2}{*}{ NOP } & \multicolumn{2}{|c|}{$\begin{array}{c}\text { Nozzle open pressure } \\
{[14-21 \mathrm{MPa}]}\end{array}$} & & \\
\hline & \multicolumn{2}{|c|}{23} & & \\
\hline Test & \multicolumn{2}{|c|}{ Injection dosage } & \multicolumn{2}{|c|}{ Return dosage } \\
\hline $\begin{array}{l}\text { Leak test }\left[\mathrm{mm}^{3} / \mathrm{H}\right] \\
170 \mathrm{MPa}, 300 \mathrm{~s}\end{array}$ & \multicolumn{2}{|c|}{0} & $0-80$ & 48.73 \\
\hline $\begin{array}{l}\text { IVM } 1\left[\mathrm{~mm}^{3} / \mathrm{H}\right] \\
160 \mathrm{MPa}, 800 \mu \mathrm{s}\end{array}$ & $41.16-52.4$ & 42.63 & $5-82$ & 63,23 \\
\hline $\begin{array}{l}\text { IVM } 2\left[\mathrm{~mm}^{3} / \mathrm{H}\right] \\
60 \mathrm{MPa}, 630 \mu \mathrm{s}\end{array}$ & $8-15.68$ & 8.14 & & \\
\hline $\begin{array}{l}\text { IVM } 3\left[\mathrm{~mm}^{3} / \mathrm{H}\right] \\
30 \mathrm{MPa}, 640 \mu \mathrm{s}\end{array}$ & $0.88-6.48$ & 1.05 & & \\
\hline $\begin{array}{l}\text { IVM } 4\left[\mathrm{~mm}^{3} / \mathrm{H}\right] \\
60 \mathrm{MPa}, 275 \mu \mathrm{s}\end{array}$ & $0.24-2.96$ & 0.11 & & \\
\hline
\end{tabular}

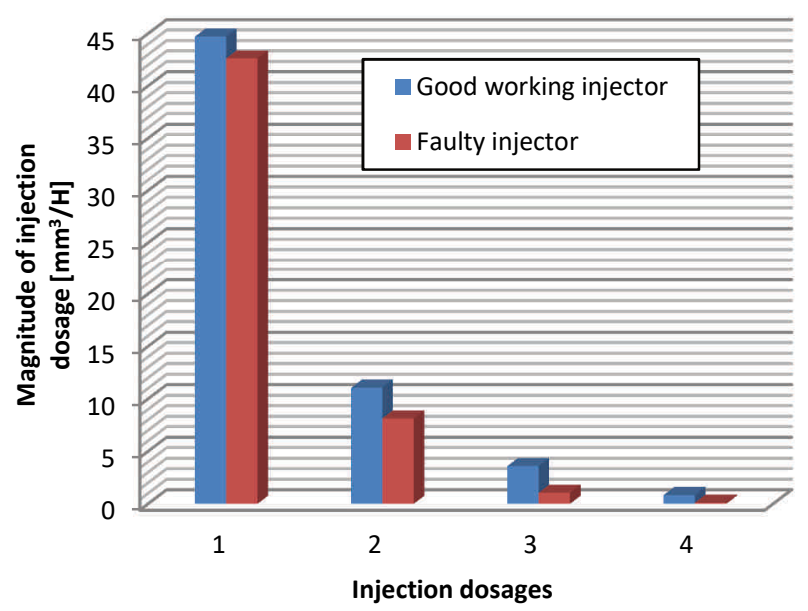

Fig. 12. Injection dosages comparison between faultless and faulty electromagnetic Bosch fuel injector; 1 - full load dosage, 2 - medium load dosage, 3 - idle speed dosage, 4 - initial dosage
Table 3. Test results of good working piezoelectric Bosch fuel injector research

\begin{tabular}{|c|c|c|c|c|}
\hline \multicolumn{5}{|c|}{ Piezoelectric Bosch fuel injector no. 0445116024} \\
\hline \multirow[t]{2}{*}{ eRLC } & \multicolumn{2}{|c|}{$\begin{array}{c}\text { Resistance } \\
{[175-200 \mathrm{k} \Omega]}\end{array}$} & \multicolumn{2}{|c|}{$\begin{array}{c}\text { Capacitance } \\
{[1.80-5.60 \mu \mathrm{F}]}\end{array}$} \\
\hline & \multicolumn{2}{|c|}{182} & \multicolumn{2}{|c|}{2.30} \\
\hline \multirow[t]{2}{*}{ RSP } & \multicolumn{2}{|c|}{$\begin{array}{l}\text { Injection delay } \\
{[100-420 \mu \mathrm{s}]}\end{array}$} & & \\
\hline & \multicolumn{2}{|c|}{208} & & \\
\hline \multirow[t]{2}{*}{ NOP } & \multicolumn{2}{|c|}{$\begin{array}{c}\text { Nozzle open pressure } \\
{[12-19 \mathrm{MPa}]}\end{array}$} & & \\
\hline & \multicolumn{2}{|c|}{14} & & \\
\hline Test & \multicolumn{2}{|c|}{ Injection dosage } & \multicolumn{2}{|c|}{ Return dosage } \\
\hline $\begin{array}{l}\text { Leak test }\left[\mathrm{mm}^{3} / \mathrm{H}\right] \\
160 \mathrm{MPa}, 85 \mathrm{~s}\end{array}$ & \multicolumn{2}{|l|}{0} & $0-1$ & 0.33 \\
\hline $\begin{array}{l}\text { IVM } 1\left[\mathrm{~mm}^{3} / \mathrm{H}\right] \\
180 \mathrm{MPa}, 800 \mu \mathrm{s}\end{array}$ & $36.10-44.25$ & 39.17 & $2-18$ & 9,18 \\
\hline $\begin{array}{l}\text { IVM } 2\left[\mathrm{~mm}^{3} / \mathrm{H}\right] \\
80 \mathrm{MPa}, 190 \mu \mathrm{s}\end{array}$ & $0.24-2.16$ & 1.22 & & \\
\hline $\begin{array}{l}\text { IVM } 3\left[\mathrm{~mm}^{3} / \mathrm{H}\right] \\
30 \mathrm{MPa}, 535 \mu \mathrm{s}\end{array}$ & $1.68-5.20$ & 4.27 & & \\
\hline $\begin{array}{l}\text { IVM } 4\left[\mathrm{~mm}^{3} / \mathrm{H}\right] \\
80 \mathrm{MPa}, 490 \mu \mathrm{s}\end{array}$ & $12.24-18.00$ & 16.31 & & \\
\hline
\end{tabular}

Table 4. Test results of faulty piezoelectric Bosch fuel injector research

\begin{tabular}{|c|c|c|c|c|}
\hline \multicolumn{5}{|c|}{ Piezoelectric Bosch fuel injector no. 0445116024} \\
\hline \multirow[t]{2}{*}{ eRLC } & \multicolumn{2}{|c|}{$\begin{array}{c}\text { Resistance } \\
{[175-200 \mathrm{k} \Omega]}\end{array}$} & \multicolumn{2}{|c|}{$\begin{array}{c}\text { Capacitance } \\
{[1.80-5.60 \mu \mathrm{F}]}\end{array}$} \\
\hline & \multicolumn{2}{|c|}{186} & \multicolumn{2}{|c|}{2.98} \\
\hline \multirow[t]{2}{*}{ RSP } & \multicolumn{2}{|c|}{$\begin{array}{c}\text { Injection delay } \\
{[100-420 \mu \mathrm{s}]}\end{array}$} & & \\
\hline & \multicolumn{2}{|c|}{387} & & \\
\hline \multirow[t]{2}{*}{ NOP } & \multicolumn{2}{|c|}{$\begin{array}{c}\text { Nozzle open pressure } \\
{[12-19 \mathrm{MPa}]}\end{array}$} & & \\
\hline & \multicolumn{2}{|c|}{23} & & \\
\hline Test & \multicolumn{2}{|c|}{ Injection dosage } & \multicolumn{2}{|c|}{ Return dosage } \\
\hline $\begin{array}{l}\text { Leak test }\left[\mathrm{mm}^{3} / \mathrm{H}\right] \\
160 \mathrm{MPa}, 85 \mathrm{~s}\end{array}$ & \multicolumn{2}{|c|}{0} & $0-1$ & 12.54 \\
\hline $\begin{array}{l}\text { IVM } 1\left[\mathrm{~mm}^{3} / \mathrm{H}\right] \\
180 \mathrm{MPa}, 800 \mu \mathrm{s}\end{array}$ & $36.10-44.25$ & 42.64 & $2-18$ & 34,75 \\
\hline $\begin{array}{l}\text { IVM } 2\left[\mathrm{~mm}^{3} / \mathrm{H}\right] \\
80 \mathrm{MPa}, 190 \mu \mathrm{s}\end{array}$ & $0.24-2.16$ & 0.34 & & \\
\hline $\begin{array}{l}\text { IVM } 3\left[\mathrm{~mm}^{3} / \mathrm{H}\right] \\
30 \mathrm{MPa}, 535 \mu \mathrm{s}\end{array}$ & $1.68-5.20$ & 1.84 & & \\
\hline $\begin{array}{l}\text { IVM } 4\left[\mathrm{~mm}^{3} / \mathrm{H}\right] \\
80 \mathrm{MPa}, 490 \mu \mathrm{s}\end{array}$ & $12.24-18.00$ & 9.34 & & \\
\hline
\end{tabular}

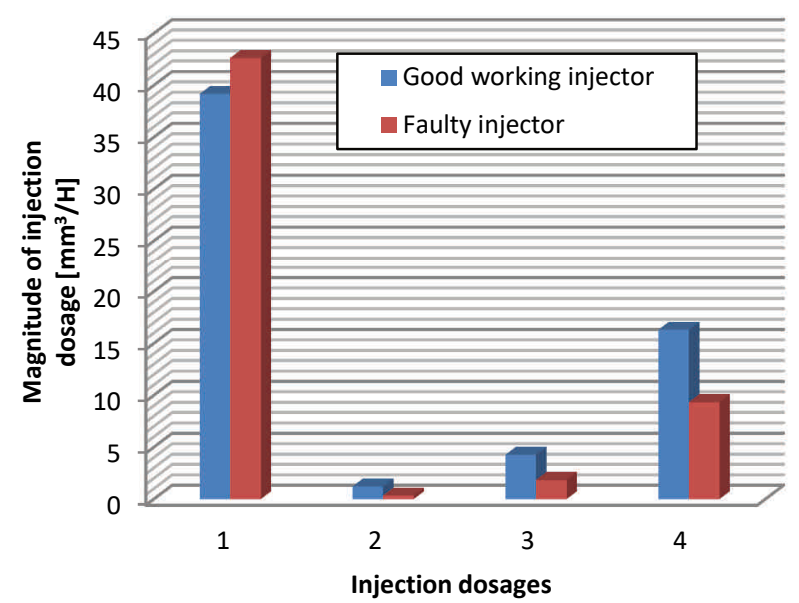

Fig. 13. Injection dosages comparison between faultless and faulty piezoelectric Bosch fuel injector 1 - full load dosage, 2 - initial dosage, 3 - idle speed dosage, 4 - medium load dosage 
Table 5. Test results of faultless electromagnetic Denso fuel injector research

\begin{tabular}{|c|c|c|c|c|}
\hline \multicolumn{5}{|c|}{ Electromagnetic Denso fuel injector no. $095000-758$} \\
\hline \multirow[t]{2}{*}{ eRLC } & \multicolumn{2}{|c|}{$\begin{array}{l}\text { Resistance } \\
{[0.4-3.8 \Omega]}\end{array}$} & \multicolumn{2}{|c|}{$\begin{array}{c}\text { Inductance } \\
{[300-4000 \mu \mathrm{H}]}\end{array}$} \\
\hline & \multicolumn{2}{|c|}{1.20} & \multicolumn{2}{|c|}{570} \\
\hline \multirow[t]{2}{*}{ RSP } & \multicolumn{2}{|c|}{$\begin{array}{l}\text { Injection delay } \\
{[390-680 \mu \mathrm{s}]}\end{array}$} & & \\
\hline & \multicolumn{2}{|c|}{605} & & \\
\hline \multirow[t]{2}{*}{ NOP } & \multicolumn{2}{|c|}{$\begin{array}{c}\text { Nozzle open pressure } \\
{[12-28 \mathrm{MPa}]}\end{array}$} & & \\
\hline & \multicolumn{2}{|c|}{21} & & \\
\hline Test & \multicolumn{2}{|c|}{ Injection dosage } & \multicolumn{2}{|c|}{ Return dosage } \\
\hline $\begin{array}{l}\text { Leak test }\left[\mathrm{mm}^{3} / \mathrm{H}\right] \\
170 \mathrm{MPa}, 45 \mathrm{~s}\end{array}$ & \multicolumn{2}{|c|}{0} & $0-60.8$ & 25.48 \\
\hline $\begin{array}{l}\text { IVM } 1\left[\mathrm{~mm}^{3} / \mathrm{H}\right] \\
160 \mathrm{MPa}, 1080 \mu \mathrm{s}\end{array}$ & $36.27-53.33$ & 49.37 & $0-69$ & 35,18 \\
\hline $\begin{array}{l}\text { IVM } 2\left[\mathrm{~mm}^{3} / \mathrm{H}\right] \\
80 \mathrm{MPa}, 760 \mu \mathrm{s}\end{array}$ & $12-20$ & 16.47 & & \\
\hline $\begin{array}{l}\text { IVM } 3\left[\mathrm{~mm}^{3} / \mathrm{H}\right] \\
25 \mathrm{MPa}, 820 \mu \mathrm{s}\end{array}$ & $2.67-6.4$ & 3.48 & & \\
\hline $\begin{array}{l}\text { IVM } 4\left[\mathrm{~mm}^{3} / \mathrm{H}\right] \\
80 \mathrm{MPa}, 400 \mu \mathrm{s}\end{array}$ & $0.8-3.47$ & 2.17 & & \\
\hline
\end{tabular}

Table 6. Test results of faulty electromagnetic Denso fuel injector research

\begin{tabular}{|c|c|c|c|c|}
\hline \multicolumn{5}{|c|}{ Electromagnetic Denso fuel injector no. $095000-758$} \\
\hline eRLC & \multicolumn{2}{|c|}{$\begin{array}{c}\text { Resistance } \\
{[0.4-3.8 \Omega]}\end{array}$} & \multicolumn{2}{|c|}{$\begin{array}{c}\text { Inductance } \\
{[300-4000 \mu \mathrm{H}]}\end{array}$} \\
\hline & \multicolumn{2}{|c|}{1.39} & \multicolumn{2}{|c|}{465} \\
\hline RSP & \multicolumn{2}{|c|}{$\begin{array}{c}\text { Injection delay } \\
{[390-680 \mu \mathrm{s}]}\end{array}$} & & \\
\hline & \multicolumn{2}{|c|}{710} & & \\
\hline \multirow[t]{2}{*}{ NOP } & \multicolumn{2}{|c|}{$\begin{array}{c}\text { Nozzle open pressure } \\
{[12-28 \mathrm{MPa}]}\end{array}$} & & \\
\hline & \multicolumn{2}{|c|}{26} & & \\
\hline Test & \multicolumn{2}{|c|}{ Injection dosage } & \multicolumn{2}{|c|}{ Return dosage } \\
\hline $\begin{array}{l}\text { Leak test }\left[\mathrm{mm}^{3} / \mathrm{H}\right] \\
170 \mathrm{MPa} .45 \mathrm{~s}\end{array}$ & \multicolumn{2}{|c|}{0} & $0-60.8$ & 48.62 \\
\hline $\begin{array}{l}\text { IVM } 1\left[\mathrm{~mm}^{3} / \mathrm{H}\right] \\
160 \mathrm{MPa}, 1080 \mu \mathrm{s}\end{array}$ & $36.27-53.33$ & 41.97 & $0-69$ & 75,08 \\
\hline $\begin{array}{l}\text { IVM } 2\left[\mathrm{~mm}^{3} / \mathrm{H}\right] \\
80 \mathrm{MPa}, 760 \mu \mathrm{s}\end{array}$ & $12-20$ & 12.94 & & \\
\hline $\begin{array}{l}\text { IVM } 3\left[\mathrm{~mm}^{3} / \mathrm{H}\right] \\
25 \mathrm{MPa}, 820 \mu \mathrm{s}\end{array}$ & $2.67-6.4$ & 2.15 & & \\
\hline $\begin{array}{l}\text { IVM } 4\left[\mathrm{~mm}^{3} / \mathrm{H}\right] \\
80 \mathrm{MPa}, 400 \mu \mathrm{s}\end{array}$ & $0.8-3.47$ & 0.38 & & \\
\hline
\end{tabular}

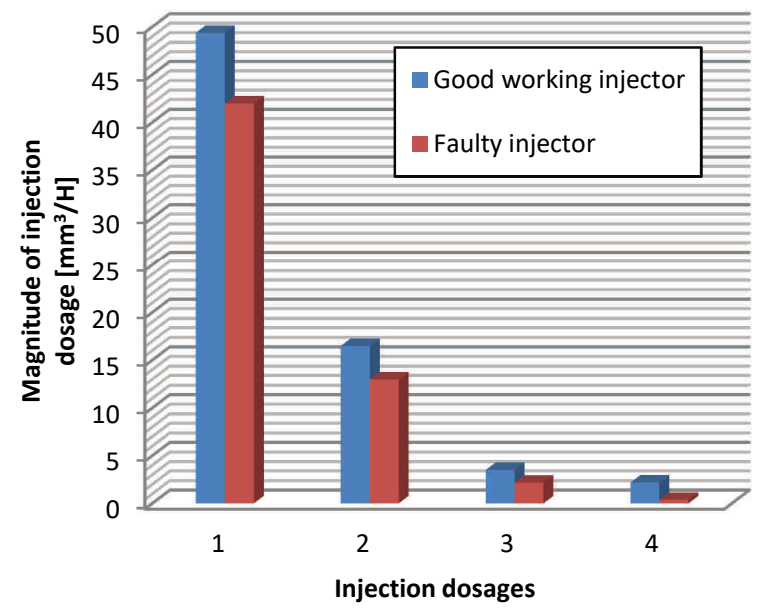

Fig. 14. Injection dosages comparison between faultless and faulty electromagnetic Denso fuel injector 1 - full load dosage, 2 - medium load dosage, 3 - idle speed dosage, 4 - initial dosage
Table 7. Test results of faultless electromagnetic Delphi fuel injector research

\begin{tabular}{|c|c|c|c|c|}
\hline \multicolumn{5}{|c|}{ Electromagnetic Delphi fuel injector no. EJBR - 02301Z } \\
\hline \multirow[t]{2}{*}{ eRLC } & \multicolumn{2}{|c|}{$\begin{array}{c}\text { Resistance } \\
{[0.2-0.6 \Omega]}\end{array}$} & \multicolumn{2}{|c|}{$\begin{array}{c}\text { Inductance } \\
{[60-110 \mu \mathrm{H}]}\end{array}$} \\
\hline & \multicolumn{2}{|c|}{0.40} & \multicolumn{2}{|c|}{87} \\
\hline \multirow[t]{2}{*}{ RSP } & \multicolumn{2}{|c|}{$\begin{array}{l}\text { Injection delay } \\
{[300-495 \mu \mathrm{s}]}\end{array}$} & & \\
\hline & \multicolumn{2}{|l|}{427} & & \\
\hline \multirow[t]{2}{*}{ NOP } & \multicolumn{2}{|c|}{$\begin{array}{c}\text { Nozzle open pressure } \\
{[13-23 \mathrm{MPa}]}\end{array}$} & & \\
\hline & \multicolumn{2}{|l|}{13} & & \\
\hline Test & \multicolumn{2}{|c|}{ Injection dosage } & \multicolumn{2}{|c|}{ Return dosage } \\
\hline $\begin{array}{l}\text { Leak test }\left[\mathrm{mm}^{3} / \mathrm{H}\right] \\
140 \mathrm{MPa}, 120 \mathrm{~s}\end{array}$ & \multicolumn{2}{|l|}{0} & $0-40$ & 2.02 \\
\hline $\begin{array}{l}\text { IVM } 1\left[\mathrm{~mm}^{3} / \mathrm{H}\right] \\
140 \mathrm{MPa}, 700 \mu \mathrm{s}\end{array}$ & $31.83-46.33$ & 38.75 & $0-40$ & 18,50 \\
\hline $\begin{array}{l}\text { IVM } 2\left[\mathrm{~mm}^{3} / \mathrm{H}\right] \\
80 \mathrm{MPa}, 600 \mu \mathrm{s}\end{array}$ & $14.43-31.35$ & 22.03 & & \\
\hline $\begin{array}{l}\text { IVM } 3\left[\mathrm{~mm}^{3} / \mathrm{H}\right] \\
23 \mathrm{MPa}, 573 \mu \mathrm{s}\end{array}$ & $0.3-5.55$ & 2.84 & & \\
\hline $\begin{array}{l}\text { IVM } 4\left[\mathrm{~mm}^{3} / \mathrm{H}\right] \\
40 \mathrm{MPa}, 462 \mu \mathrm{s}\end{array}$ & $1.18-7.91$ & 4.69 & & \\
\hline
\end{tabular}

Table 8. Test results of faulty electromagnetic Delphi fuel injector research

\begin{tabular}{|c|c|c|c|c|}
\hline \multicolumn{5}{|c|}{ Electromagnetic Delphi fuel injector no. EJBR - 02301Z } \\
\hline eRLC & \multicolumn{2}{|c|}{$\begin{array}{c}\text { Resistance } \\
{[0.2-0.6 \Omega]}\end{array}$} & \multicolumn{2}{|c|}{$\begin{array}{c}\text { Inductance } \\
{[60-110 \mu \mathrm{H}]}\end{array}$} \\
\hline & \multicolumn{2}{|c|}{0.35} & \multicolumn{2}{|c|}{74} \\
\hline RSP & \multicolumn{2}{|c|}{$\begin{array}{c}\text { Injection delay } \\
{[300-495 \mu \mathrm{s}]}\end{array}$} & & \\
\hline & \multicolumn{2}{|c|}{475} & & \\
\hline \multirow[t]{2}{*}{ NOP } & \multicolumn{2}{|c|}{$\begin{array}{c}\text { Nozzle open pressure } \\
{[13-23 \mathrm{MPa}]}\end{array}$} & & \\
\hline & \multicolumn{2}{|c|}{17} & & \\
\hline Test & \multicolumn{2}{|c|}{ Injection dosage } & \multicolumn{2}{|c|}{ Return dosage } \\
\hline $\begin{array}{l}\text { Leak test }\left[\mathrm{mm}^{3} / \mathrm{H}\right] \\
140 \mathrm{MPa}, 120 \mathrm{~s}\end{array}$ & \multicolumn{2}{|l|}{0} & $0-40$ & 34.97 \\
\hline $\begin{array}{l}\text { IVM } 1\left[\mathrm{~mm}^{3} / \mathrm{H}\right] \\
140 \mathrm{MPa}, 700 \mu \mathrm{s}\end{array}$ & $31.83-46.33$ & 42.29 & $0-40$ & 55,18 \\
\hline $\begin{array}{l}\text { IVM } 2\left[\mathrm{~mm}^{3} / \mathrm{H}\right] \\
80 \mathrm{MPa}, 600 \mu \mathrm{s}\end{array}$ & $14.43-31.35$ & 15.86 & & \\
\hline $\begin{array}{l}\text { IVM } 3\left[\mathrm{~mm}^{3} / \mathrm{H}\right] \\
23 \mathrm{MPa}, 573 \mu \mathrm{s}\end{array}$ & $0.3-5.55$ & 0.64 & & \\
\hline $\begin{array}{l}\text { IVM } 4\left[\mathrm{~mm}^{3} / \mathrm{H}\right] \\
40 \mathrm{MPa}, 462 \mu \mathrm{s}\end{array}$ & $1.18-7.91$ & 2.12 & & \\
\hline
\end{tabular}

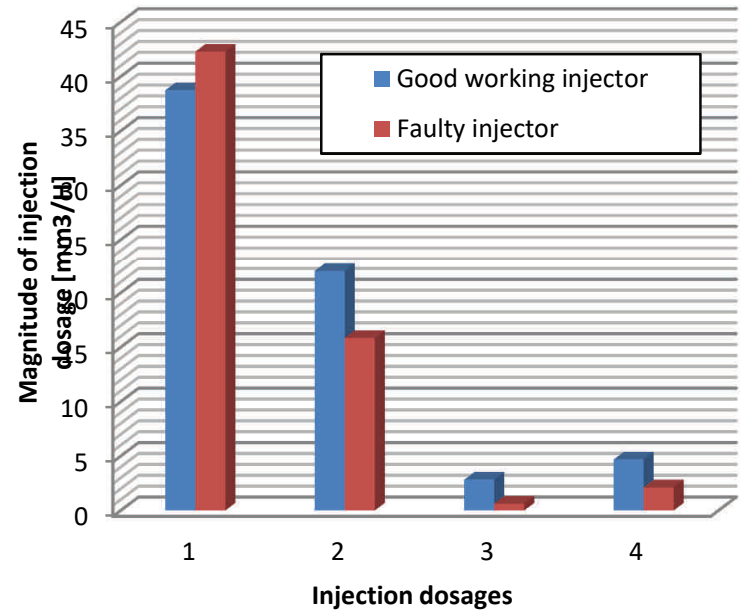

Fig. 15. Injection dosages comparison between faultless and faulty electromagnetic Delphi fuel injector 1 - full load dosage, 2 - medium load dosage, 3 - idle speed dosage, 4 - initial dosage 
Table 9. Test results of faultless piezoelectric Continental fuel injector research

\begin{tabular}{|c|c|c|c|c|}
\hline \multicolumn{5}{|c|}{ Piezoelectric Continental fuel injector no. A2C59511603 } \\
\hline eRLC & \multicolumn{2}{|c|}{$\begin{array}{c}\text { Resistance } \\
{[175-200 \mathrm{k} \Omega]}\end{array}$} & \multicolumn{2}{|c|}{$\begin{array}{c}\text { Capacitance } \\
{[2.20-7.10 \mu \mathrm{F}]}\end{array}$} \\
\hline & \multicolumn{2}{|c|}{187} & \multicolumn{2}{|c|}{3.35} \\
\hline RSP & \multicolumn{2}{|c|}{$\begin{array}{l}\text { Injection delay } \\
{[200-420 \mu \mathrm{s}]}\end{array}$} & & \\
\hline & \multicolumn{2}{|l|}{257} & & \\
\hline \multirow[t]{2}{*}{ NOP } & \multicolumn{2}{|c|}{$\begin{array}{c}\text { Nozzle open pressure } \\
{[12-19 \mathrm{MPa}]}\end{array}$} & & \\
\hline & \multicolumn{2}{|l|}{15} & & \\
\hline Test & \multicolumn{2}{|c|}{ Injection dosage } & \multicolumn{2}{|c|}{ Return dosage } \\
\hline $\begin{array}{l}\text { Leak test }\left[\mathrm{mm}^{3} / \mathrm{H}\right] \\
160 \mathrm{MPa}, 180 \mathrm{~s}\end{array}$ & \multicolumn{2}{|l|}{0} & $0-65$ & 4.31 \\
\hline $\begin{array}{l}\text { IVM } 1\left[\mathrm{~mm}^{3} / \mathrm{H}\right] \\
160 \mathrm{MPa}, 1100 \mu \mathrm{s}\end{array}$ & $55.45-67.45$ & 61.15 & $0-40$ & 10,19 \\
\hline $\begin{array}{l}\text { IVM } 2\left[\mathrm{~mm}^{3} / \mathrm{H}\right] \\
70 \mathrm{MPa}, 380 \mu \mathrm{s}\end{array}$ & $10.82-15.82$ & 13.82 & & \\
\hline $\begin{array}{l}\text { IVM } 3\left[\mathrm{~mm}^{3} / \mathrm{H}\right] \\
70 \mathrm{MPa}, 140 \mu \mathrm{s}\end{array}$ & $0.5-4.51$ & 2.79 & & \\
\hline $\begin{array}{l}\text { IVM } 4\left[\mathrm{~mm}^{3} / \mathrm{H}\right] \\
25 \mathrm{MPa}, 520 \mu \mathrm{s}\end{array}$ & $3.28-8.28$ & 6.17 & & \\
\hline
\end{tabular}

Table 10. Test results of faulty piezoelectric Continental fuel injector research

\begin{tabular}{|c|c|c|c|c|}
\hline \multicolumn{5}{|c|}{ Piezoelectric Continental fuel injector no. A2C59511603 } \\
\hline eRLC & \multicolumn{2}{|c|}{$\begin{array}{c}\text { Resistance } \\
{[175-200 \mathrm{k} \Omega]}\end{array}$} & \multicolumn{2}{|c|}{$\begin{array}{c}\text { Capacitance } \\
{[2.20-7.10 \mu \mathrm{F}]}\end{array}$} \\
\hline & \multicolumn{2}{|c|}{178} & \multicolumn{2}{|c|}{2.96} \\
\hline \multirow[t]{2}{*}{ RSP } & \multicolumn{2}{|c|}{$\begin{array}{l}\text { Injection delay } \\
{[200-420 \mu \mathrm{s}]}\end{array}$} & & \\
\hline & \multicolumn{2}{|c|}{643} & & \\
\hline \multirow[t]{2}{*}{ NOP } & \multicolumn{2}{|c|}{$\begin{array}{c}\text { Nozzle open pressure } \\
{[12-19 \mathrm{MPa}]}\end{array}$} & & \\
\hline & \multicolumn{2}{|c|}{21} & & \\
\hline Test & \multicolumn{2}{|c|}{ Injection dosage } & \multicolumn{2}{|c|}{ Return dosage } \\
\hline $\begin{array}{l}\text { Leak test }\left[\mathrm{mm}^{3} / \mathrm{H}\right] \\
160 \mathrm{MPa}, 180 \mathrm{~s}\end{array}$ & \multicolumn{2}{|c|}{0} & $0-65$ & 18.53 \\
\hline $\begin{array}{l}\text { IVM } 1\left[\mathrm{~mm}^{3} / \mathrm{H}\right] \\
160 \mathrm{MPa}, 1100 \mu \mathrm{s}\end{array}$ & $55.45-67.45$ & 56.31 & $0-40$ & 27,92 \\
\hline $\begin{array}{l}\text { IVM } 2\left[\mathrm{~mm}^{3} / \mathrm{H}\right] \\
70 \mathrm{MPa}, 380 \mu \mathrm{s}\end{array}$ & $10.82-15.82$ & 8.53 & & \\
\hline $\begin{array}{l}\text { IVM } 3\left[\mathrm{~mm}^{3} / \mathrm{H}\right] \\
70 \mathrm{MPa}, 140 \mu \mathrm{s}\end{array}$ & $0.5-4.51$ & 0.65 & & \\
\hline $\begin{array}{l}\text { IVM } 4\left[\mathrm{~mm}^{3} / \mathrm{H}\right] \\
25 \mathrm{MPa}, 520 \mu \mathrm{s}\end{array}$ & $3.28-8.28$ & 2.76 & & \\
\hline
\end{tabular}

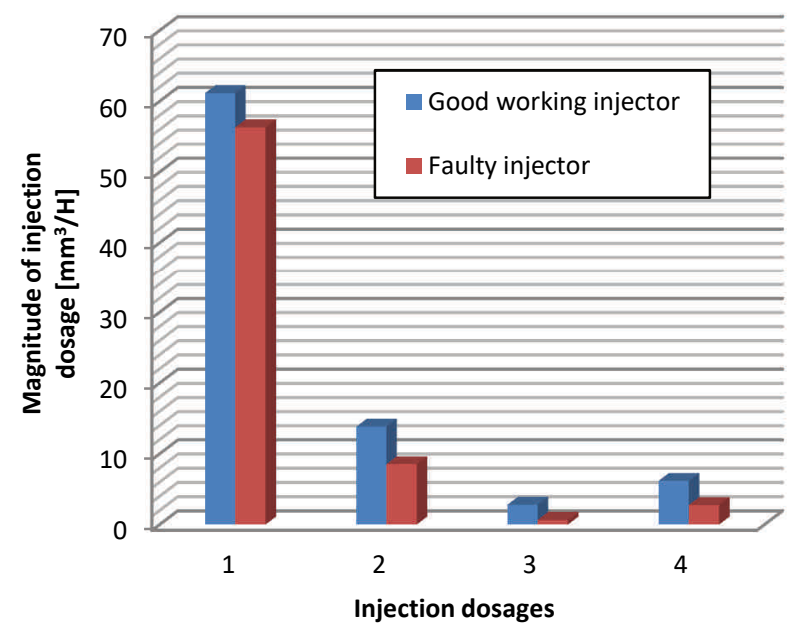

Fig. 16. Injection dosages comparison between faultless and faulty piezoelectric Continental fuel injector 1 - full load dosage, 2 -medium load dosage, 3 - initial dosage, 4 - idle speed dosage

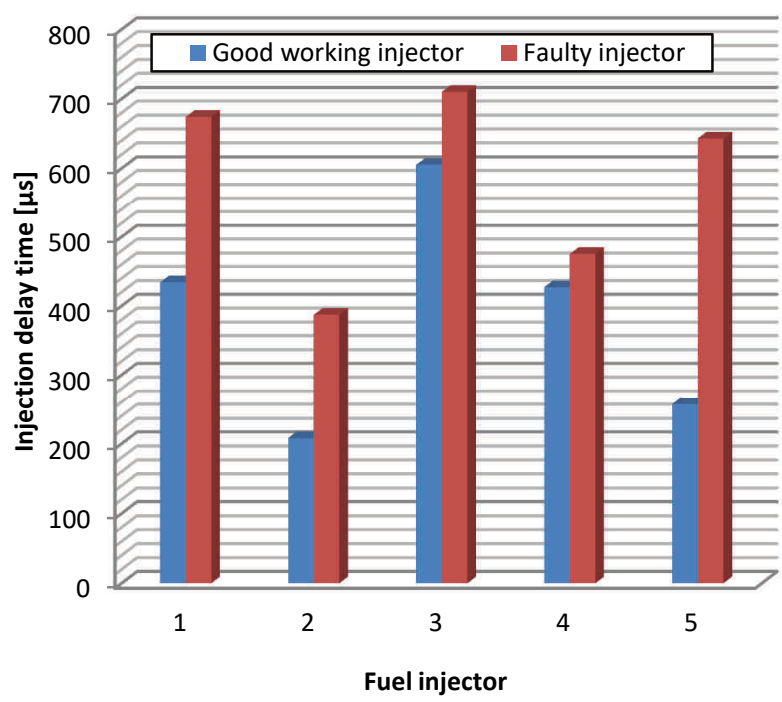

Fig. 17. Comparison of RSP parameter between faultless and faulty fuel injectors 1 - electromagnetic Bosch fuel injector, 2 - piezoelectric Bosch fuel injector, 3 - electromagnetic Denso fuel injector, 4 - electromagnetic

Delphi fuel injector, 5 - piezoelectric Continental fuel injector

Tables 1-10 present the results of tests of the number of different fuel injectors. Tests have been made in the fuel injections systems diagnostic and repair laboratory using the test bench Carbon Tech CRU 2i. Main fuel injectors parameters like injection and return dosages, electric values, nozzle open pressure and injection delay (RSP) were measured.

Figures 12-16 present results of fuel injector dosages tests for a set of fuel injectors under different operational conditions, i.e. full load, medium load, idle speed and initial dosages. It is evident that in all the cases corrosion resulted in decreased fuel dosages, what is especially visible for idle-speed tests. Corrosion pollution change work characteristic of injector needle in the nozzle. Figure 17 presents results of RSP parameter measurements. Injection delay is important indicator, which depends on injector construction. The fastest are piezoelectric fuel injectors. Too long injection delays influence on combustion process.

\section{Conclusion}

Corrosion phenomena is a process which slowly damages metal elements. There are a few types of corrosion processes in fuel injectors. This problem should be examined in two aspects: when liquid is in rest or when it is in move. Fuel injector parts wore out during operation because of erosion, cavitation and friction. The conditions like high pressure, high temperature, poor fuel quality (its pollution and humidity) intensify corrosion. All fuel injectors are made with the same material and magnitude of corrosion depends on medium not on the type of injector. Extensive and deep corrosion pits on precision elements result in loss of fuel tightness. Results of tests of various fuel injectors (Table 1-10) show that corrosion phenomena influences fuel injectors parameters. Corroded precision elements (Figs 6-8) increase leaks what affects return dosage. High inner leaks may lead to unsealing of the whole injection system. Corroded nozzle with idle and steering valve af- 
fects injector operational parameters like: injection dosages, nozzle open pressure and injection delay.

Electromagnetic and piezoelectric fuel injectors have been tested and analyzed. Corrosion phenomena has similar effect on both of them. The tests of modern fuel injectors consist of checking injection and return dosages. If precision elements are damaged return dosages increase, what makes injector valve and nozzle very important. Corrosion, cavitation and friction trigger damaging phenomenon which slowly destroys them. The first sign of injector failure is loss of micro dosages when operating in low pressure, short injection time conditions (e.g. $25 \mathrm{MPa}$ and $250 \mu \mathrm{s}$ ).

The results of corrosion phenomena are decrease of initial dosages especial for idle speed and medium engine load.

Idle speed dosage is very important as fuel injector diagnostic parameter. If the differences between idle speed dosages are higher than $0.5 \mathrm{~mm}^{3} / \mathrm{H}$ then the fuel injector's elements are already worn and the electronic diagnostics is able to notice this deterioration. The corrosion pollution change range of movement injector idle in the nozzle, what has an impact on injection dosages and nozzle open pressure (NOP). If corrosion pits aren't extensive and deep (Fig. 11) it should be possible to repair injector. The repair involves very accurate cleaning in ultrasonic washers and regulation. When damage is extensive it is necessary to replace elements. Another very important fuel injector operational parameter is injection delay time (RSP). This is the time between electric steering fuel injector and when it starts spraying. Piezoelectric fuel injectors have the shortest delay. Short RSP enables generation of more injections during a single cycle. Too long injection delay compounds combustion process what influences on engine work. Corroded injector components extend injection delay.

\section{Nomenclature}

CI compression ignition

CR common rail system

\section{Bibliography}

[1] AMBrozIK, A., AMBrozIK, T., ŁAGOWSKI, P. Fuel impact on emissions of harmful components of the exhaust gas from the CI engine during cold start up. Eksploatacja $i$ Niezawodnosc - Maintenance and Realiability. 2015, 17(1), 95-99.

[2] BASZKIEWICZ, J., KAMIŃSKI, M. Korozja materiałów. Oficyna Wydawnicza Politechniki Warszawskiej. 2006.

[3] GÜNTHER, H. Common Rail - Systeme in der Werkstattpraxis. Technik, Prüfung, Diagnose. Bad Wörihofen: Krafthand Verlag Walter Schultz GmbH. 2012.

[4] HRYNIEWICZ, T., ROKOSZ, K. Podstawy teoretyczne i aspekty praktyczne zjawiska korozji. Wydawnictwo Uczelniane Politechniki Koszalińskiej. 2010.

Tomasz Osipowicz, DEng. - Faculty of Mechanical Engineering and Mechatronics at West Pomeranian University of Technology.

e-mail: Tomasz.Osipowicz@zut.edu.pl
[5] Zasobnikowe układy wtryskowe Common Rail. Informator Techniczny Sterowanie silników ZS. Robert Bosch GMBH. WKiŁ. 2005, 2009.

[6] OSIPOWICZ, T., ABRAMEK, K.F., STOECK, T. Testing of modern common rail fuel injectors. Combustion Engines. 2015, 162(3), 688-694.

[7] OSIPOWICZ, T. Diagnosing Common Rail fuel injectors using fuel micro - doses. Teka. Commission of Motorization and Energetics in Agriculture. 2015, 15(1), 61-64.

[8] OSIPOWICZ, T. Diagnosis Diesel Common Rail fuel systems. Autobusy, Technika, Eksploatacja, Systemy Transportowe. 2016, 8, 129-130.

Maciej Lisowski, DSc., DEng. - Faculty of Mechanical Engineering and Mechatronics at West Pomeranian University of Technology.

e-mail:MLisowski@zut.edu.pl 